\title{
Detection of Pulsed X-ray Emission from The Fastest Millisecond Pulsar PSR B1937+21 with ASCA
}

\author{
Motoki Takahashi ${ }^{1}$, Shinpei Shibata ${ }^{1}$, Ken'ichi Torii ${ }^{2}$, Yoshitaka Saito ${ }^{3}$, \\ Nobuyuki Kawai ${ }^{2,4}$, Masaharu Hirayama ${ }^{5}$, Tadayasu Dotani ${ }^{3}$, Shuichi \\ Gunji ${ }^{1}$ and Hirohisa Sakurai ${ }^{1}$ \\ ${ }^{1}$ Department of Physics Yamagata University, Kojirakawa, Yamagata \\ 990-8560, Japan \\ ${ }^{2}$ NASDA TKSC SURP, 2-1-1 Sengen, Tsukuba, Ibaraki 305-8505, Japan \\ ${ }^{3}$ The Institute of Space and Astronautical Science, Sagamihara, \\ Kanagawa 229, Japan \\ ${ }^{4}$ The Institute of Physical and Chemical Research (RIKEN), Wako, \\ Saitama 351-01, Japan \\ ${ }^{5}$ Santa Cruz Institute for Particle Physics University of California, \\ Santa Cruz, CA 95064
}

\begin{abstract}
We report the first detection of the pulsed X-ray emission from the fastest millisecond pulsar known PSR B1937 $+21(P=1.558 \mathrm{msec})$ with ASCA. The pulsar is detected as a point source above $\sim 1.7 \mathrm{keV}$, with no nebulosity indicated. The source flux in the energy band $2-10 \mathrm{keV}$ is found to be $f=4.3 \times 10^{-13} \mathrm{erg} \mathrm{s}^{-1} \mathrm{~cm}^{-2}$, which corresponds to the luminosity of $L_{\mathrm{x}}=4 \pi D^{2} f \approx 6.6 \times 10^{32}(D / 3.6 \mathrm{kpc})^{2} \mathrm{erg} \mathrm{s}^{-1}$, where $D$ is the distance, and correspond to $\approx 6 \times 10^{-4}$ of the rotation power of the pulsar. The pulsation is found at the period predicted by the radio ephemerides with very narrow primary peaks, the width of which is about $1 / 16$ phase $\approx 100 \mu$ s near the time resolution limit $(61 \mu \mathrm{s})$ of the observation. The pulsed luminosity within the primary peak (1/16 phase interval) is found to be $f_{p}=4.0 \times 10^{-12} \mathrm{erg} \mathrm{s}^{-1} \mathrm{~cm}^{-2}$. Although there may be a secondary peak, its statistical significance is too low to identify. Spectra of the whole source region and the primary peak are fitted by power law models to give photon indices of about unity for both.
\end{abstract}

\section{Introduction}

The fastest millisecond pulsar PSR B1937+21 can own the strongest magnetic field at the light cylinder (l-c), which can exceeds the value of the Crab pulsar, $\sim 10^{6} \mathrm{G}$, while their surface fields $\sim 10^{8} \mathrm{G}$ are four orders of magnitude smaller than the typical values for the ordinary pulsars $\sim 10^{12} \mathrm{G}$. There are two proposed emission regions in the pulsar magnetosphere, the polar gap near the surface and the outer gap near the $\mathrm{l}-\mathrm{c}$. The field strength of the fastest millisecond pulsars is similar to the Crab value for the outer gap, and it is much smaller for the polar 
gap. Therefore, detection of the magnetospheric emission from the millisecond pulsars is certainly expected to contribute to the debate about the location of particle acceleration in the pulsar magnetosphere.

\section{Observation and Results}

The fastest millisecond pulsar known is PSR B1937+21 (Backer et al. 1982) and was observed with the ROSAT, given only an upper bound flux (Verbunt et al. 1996). We observed PSR B1937+21 with ASCA (Tanaka et al. 1994) on 15-17 November 1997 with the duration of $97,140 \mathrm{~s}$. And we detected the X-ray emission in the energy bands higher than $\sim 1.7 \mathrm{keV}$; we give the results of the spatial, temporary and spectral analysis (Takahashi et al. 1998).
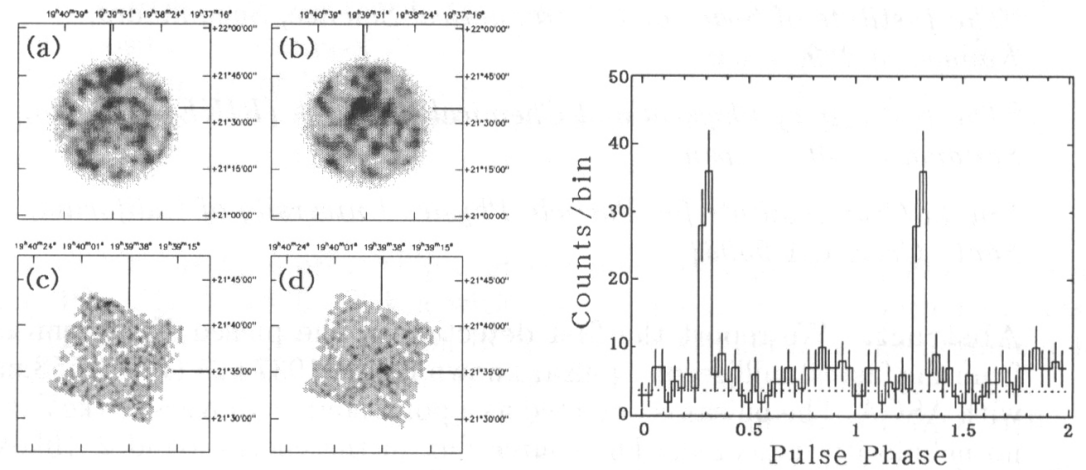

Figure 1. Left: X-ray images of PSR B1937+21 obtained with ASCA. (a) GIS Soft Band (0.7-2 keV), (b) GIS Hard Band (2-10 keV), (c) SIS Soft Band (0.5-2 keV), (d) SIS Hard Band (2-10 keV). The radio position is indicated by the vertical and horizontal lines in the images. Right: Integrated pulse profile of PSR B1937+21 in the GIS $1.7-6.5 \mathrm{keV}$ band. The dashed line indicates the background level, 3.67 counts/bin, where the 32 phase bins are used.

Acknowledgments. This work is supported in part by a Grant-in-Aid for Scientific Research (10117203) from the Ministry of Education, Science, and Culture in Japan.

\section{References}

Backer, D.C., Kulkarni, S.R., Heiles, C., Davis, M.M., \& Goss, W.M., 1982, Nature, 300, 615

Takahashi, M., Shibata, S., Torii, K., Saito, Y.\& Kawai, N., 1998, IAUC, 7030, 3

Tanaka, Y., Inoue, H. \& Holt, S. S. 1994, PASJ, 46, L37

Verbunt, F., Kuiper, L., Belloni, T., Johnston, H. M., De Bruyn, A. G., Hermsen, W., \& Van Der Klis, M., 1996, A\&A, 311, L9 